\title{
Recovery of Cometary Microorganisms from the Stratosphere
}

\section{Milton Wainwright* ${ }^{\star 1,3}$, N Chandra Wickramasinghe ${ }^{3}$, Christopher E Rose, Alexander J Baker ${ }^{2}$}

${ }^{1}$ Department of Molecular Biology and Biotechnology, University of Sheffield, UK

${ }^{2}$ Leonardo Centre for Tribology, University of Sheffield, UK

${ }^{3}$ Centre for Astrobiology, University of Buckingham, UK

\begin{abstract}
The Hoyle-Wickramasinghe theory of cometary panspermia posits that terrestrial life was introduced by comets, and predicts that this process can be tested by the detection of an ongoing incidence onto the Earth of biological entities. Searching the stratosphere for microorganisms began tentatively in the 1960's but more serious attempts to recover microorganisms from the stratosphere commenced after 2001. Since this time evidence for a continuing microbiological input from space has accumulated but such evidence has tended to be either overlooked or dismissed as contaminants. Our most recent balloon flight of July 2013 to a height of 22-27 km above Wakefield, West Yorkshire, England led to the collection of several types of microorganisms directly onto electron microscope stubs, some leading to the formation of micro-craters and so confirming infall at high speed as well as their extraterrestrial origin. In one instance a sphere of diameter $30 \mu \mathrm{m}$ was isolated and found to be mainly composed of titanium in its outer layers (with smaller amounts of vanadium). Nano-manipulation and EDX analysis showed that the titanium sphere contains a carbonaceous non-granular interior material which we suggest is a biological protoplast. Other isolates include distinctly biological filaments, a diatom frustule and a few unidentified biological entities. The relatively large sizes of the particles point decisively to their extraterrestrial origin.
\end{abstract}

Keywords: Stratospheric Sampling; Panspermia

\section{Introduction}

A prediction of the theory of cometary panspermia is that microorganisms, singly, and in clumps, as well as viruses, continue to enter the Earth from space [1-3]. An estimated total of $\sim 100$ tonnes of cometary material arrives at the Earth on a daily basis, but most of this is in the form of micrometeoroids that burn up on entry. Even if only a minute fraction of this input is in the form of microorganisms that survive entry, the process of biological ingress is at least in principle detectable, and would provide an important test of the theory of panspermia.

The extension of the terrestrial biosphere to include various levels of the atmosphere has been under discussion for several decades, particularly in relation to the transport of pathogens from one location on the Earth to another [4-6]. Microorganisms in cumulous clouds are recognised to play a role in nucleating ice crystals, but their source in most cases has been generally considered to be the surface of the Earth - terrestrial microbes carried to the troposphere in wind and air currents.

Convection currents and atmospheric turbulence leads to mixing of ground-level particulates in the air that can be carried with relative ease into the troposphere, and even lead to transport between continents on jet streams. Micro-organisms thus carried can alter the chemical composition of tropospheric clouds and induce precipitation and seeding of rain clouds $[7,8]$. However, although transport of microbes from the ground into the troposphere occurs easily, temperature inversions above heights of $\sim 15 \mathrm{~km}$ lead to barriers through which very few aerosol particles can penetrate.

Whenever rare events such as volcanic eruptions loft particles above $30 \mathrm{~km}$, particles larger than a few micrometres in radius fall back very quickly under gravity. The isothermal temperature regime between 15 and $25 \mathrm{~km}$ effectively stops the ascent of particulates, and the rapidly rising ambient temperature gradient at higher levels makes the upper stratosphere almost impervious to the transport of aerosols from ground level. It would be a reasonable assumption, therefore, to consider any microorganisms, or clumps of microorganisms, recovered from the stratosphere above $20 \mathrm{~km}$ to be of extraterrestrial origin.

\section{Earlier Attempts to RecoverStratospheric Microorganisms}

Attempts to search for microbial populations in the stratosphere were commenced immediately prior to the advent of the Space Age in the 1960s $[9,10]$. Studies carried out in this period reached altitudes of $80 \mathrm{~km}$ in some cases, and this work is reviewed in an article by Burch [11]. Bacteria as well as fungi were claimed to be found in samples collected from altitudes in the range $18-39 \mathrm{~km}$, but all these results were generally dismissed without a full assessment as being contaminants. Such a dismissal may have been partly justified in view of the primitive nature of the sterilisation and identification protocols that were available at the time.

After nearly 4 decades of inactivity on this front Narlikar [12] sought to repeat the experiments of the 1960s using rigorously sterilised cryosampler devices. In January 2001 stratospheric air with its aerosol content from $41 \mathrm{~km}$ was collected under aseptic conditions, using a balloon-borne device (cryosampler) flown over Hyderabad, India, and examined in the laboratory [13,14]. Clumps of putative bacterial colonies were discovered under SEM examination, as well as evidence of viable but non-culturable microorganisms detected by the use of fluorescent dyes, as shown in Figure 1 [13]. There were also two cultures of common microbial species with high ultraviolet resistance that were obtained by Wainwright [14]. Since these microbial species were

*Corresponding author: Milton Wainwright, Department of Molecular Biology and Biotechnology, University of Sheffield, UK, Tel: 44-1142224410. E mail: m.wainwright@sheffield.ac.uk

Received May 19, 2014; Accepted June 04, 2014; Published June 06, 2014

Citation: Wainwright M, Wickramasinghe NC, Rose CE, Baker AJ (2014) Recovery of Cometary Microorganisms from the Stratosphere. Astrobiol Outreach 2:110. doi: 10.4172/2332-2519.1000110

Copyright: (c) 2014 Wainwright M, et al. This is an open-access article distributed under the terms of the Creative Commons Attribution License, which permits unrestricted use, distribution, and reproduction in any medium, provided the original author and source are credited. 


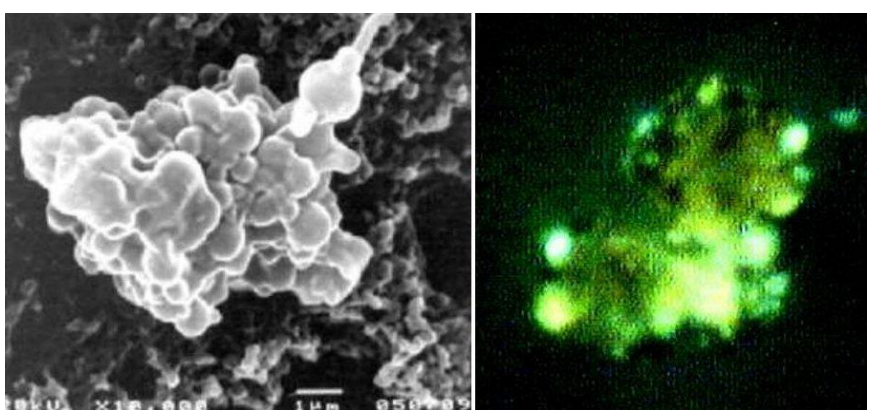

Figure 1: Left: A SEM of a colony of putative bacteria sampled from the stratosphere.

Right: Clump of cells from a stratospheric isolate fluorescing after staining with carbocyanine dye (Harris et al, 2002)

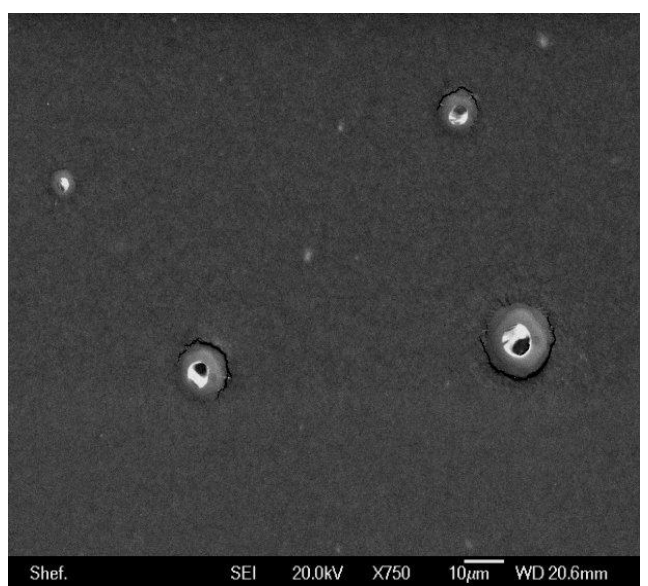

Figure 2: E/M stubs with microcrater pits caused by micrometeorites impacting from above at speed of $\mathrm{cm} / \mathrm{s}$.

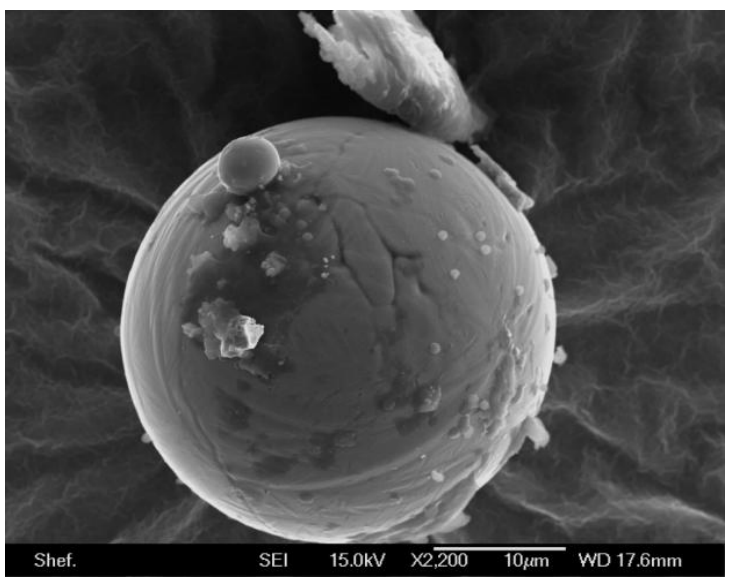

Figure 3a: Large spherical object (LSO) isolated from the stratosphere showing a smaller sphere attached and surface debris.

recovered from heights of $41 \mathrm{~km}$, significantly above the tropopause, it was inferred, not unreasonably, that they fell from above. On the basis of limited statistics that were available from the data, the daily input of cometary microorganisms was inferred to be $\sim 0.1$ tonne per day averaged over the whole Earth.
A few years after other investigators [15-17] repeated this work and recovered microbial cultures from similar heights in the stratosphere, which they attributed to be of terrestrial origin Shivaji et al [17]. Isolated 3 new bacterial strains representing novel species and gave them names with an astronomical provenance: Janibacter hoylei (sp nov), Bacillus isronensis (sp nov) and Bacillus aryabhathai (sp nov). A terrestrial origin for all three organisms was assumed (wrongly, in our view) on account of high degrees of DNA sequence homologies with known terrestrial species. Such homologies are, however, to be expected if all terrestrial microorganisms have their ultimate origins in comets, and if the evolution of life continues to be dictated by the continuing input of cometary genes $[3,18]$.

\section{July 2013 Experiments}

A balloon-launched sampling device was released from Chester, NW England on 31st July, 2013 [19]. The sampler included a drawer mechanism that could be opened and closed at a predetermined height. The stratosphere sampler carried a video camera by which the opening and closing of the sampling drawer could be viewed, confirmed and recorded. The sampling apparatus was protected from downfall of contaminating particulate matter from the balloon by a cover. Prior to launch, the inside of the draw device was scrupulously cleaned, air blasted and finally swabbed with alcohol. New scanning electron microscope stubs were placed in rows inside the drawer with their top surfaces facing outwards so that when the draw was opened any particulate matter in the stratosphere would attach to them and they could later be removed for examination under the scanning electron microscope. The protective layer on the surface of the stub was peeled off just before launching under a cover to prevent any particulate contamination. After sampling, the apparatus was transported to the laboratory and opened under conditions which avoided exposure of the stubs to contaminating dust and the stubs were similarly transferred under cover to the scanning $\mathrm{E} / \mathrm{M}$. The stubs were then sputter-coated with gold for 30 secs at $30 \mathrm{~mA}$ and then examined using a SEM (JEOL $6500 \mathrm{~F})$.

\section{Balloon launch}

The balloon was launched from an open field near Dunham on the Hill (near Ellesmere Port, Cheshire, England) during daylight hours and traversed to just south of Wakefield in West Yorkshire, England, where the samples were collected. The sampling drawer was opened for 17 minutes as the balloon rose from $22026 \mathrm{~m}$ to $27008 \mathrm{~m}$. The sampling apparatus was returned to Earth (by parachute) undamaged and intact.

A separate control flight was made to the stratosphere prior to the sampling flight, when the drawer was not opened, but all other sampling procedures were observed. No particulate matter was found (using the SEM) on any of the unexposed microscope stubs, This shows that the drawer remained airtight and that none of the stubs was exposed to particles at, or near, ground-level or at any height up to the stratosphere. These results also show that no particles contaminated the stubs during any of the sample processing procedures, thereby demonstrating that the scrupulous procedures used to prevent ground-level contamination proved effective and that no such contamination occurred.

\section{Biological Entities Discovered}

The exposed SEM stubs returned to the laboratory were examined under meticulously clean conditions. A variety of biological structures were identified, mostly lodged within larger structures with overall sizes well in excess of several micrometers. Their large sizes, combined with the remarkable fact that in some cases graphite stubs were cratered 
Citation: Wainwright M, Wickramasinghe NC, Rose CE, Baker AJ (2014) Recovery of Cometary Microorganisms from the Stratosphere. Astrobiol Outreach 2:110. doi: 10.4172/2332-2519.1000110

Page 3 of 5

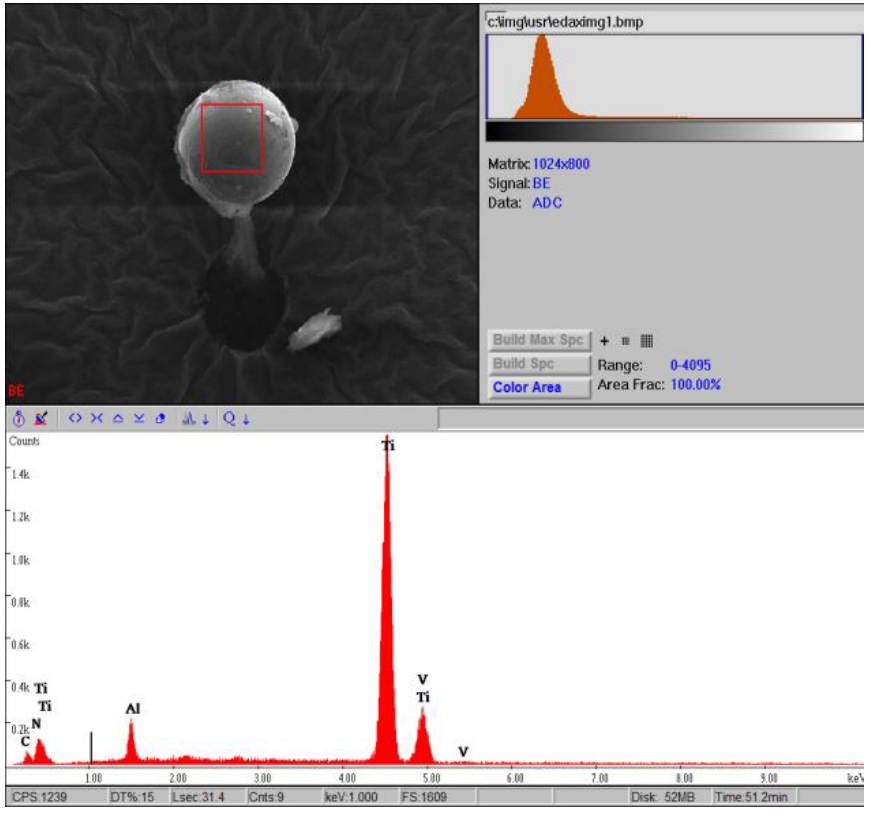

Figure 3b: EDX analysis of the LSO.

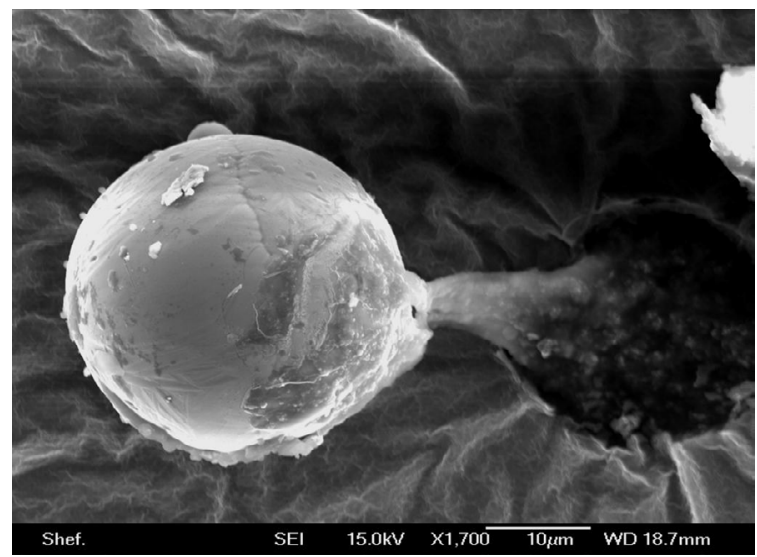

Figure 3c: Close-up of a putative biological object and material issuing from it at the point where the object was dragged from its impact site. Note damage to surface layer and underlying subsurface and smaller sphere at rear of LSO, following rotation of LSO under nanomanipulation.

in impact, provide proof that the biological entities in question arrived at speed from space and were not "lazily lifted" from Earth to the stratosphere. A sample of microcrater sites is shown in Figure 2.

One case of particular interest amongst the recovered particles is a sphere of presumed organic (carbon-rich composition) surrounded by an outer layer of titanium.

Figure $3 \mathrm{a}$ shows two spherical objects which were isolated on sampling stubs, the main, or large spherical object (LSO) having on its surface a smaller, spherical object (SSO). The central surface of the LSO is shown by EDX analysis to be mainly made up of titanium, with smaller amounts of vanadium, carbon and nitrogen (Figure $3 b)$. This structure was partially lodged in the crater and was micromanipulated out of it as seen in Figure $3 \mathrm{c}$. Here a close up of the impact crater and the attachment of the matrix material to the crater and its emergence through the hole structure is clearly seen, as well as surface damage caused to the right lower part of the putative organism. A thin amorphous surface layer can be seen and a distinct "knitted" pattern of emerging filaments, some of which bifurcate and which are clearly biological in nature.

Figure $4 \mathrm{a}$ shows another object recovered on the stubs of size around 200 micron. Long threads can be seen throughout the central part of the image, some showing signs of obvious branching; the filaments in the central region of the photograph also originate from what appears to be a mass of bacteria covered in slime material, suggesting that the filaments are bacterial. EDX analysis (Figure 4b) shows that the filaments are made of carbon and oxygen only and there are no signs of the presence of inorganic ions; particles of which might simulate biological entities. It is obvious from both Figures $4 \mathrm{a}$ and $4 \mathrm{~b}$ that the filaments show signs of flattening, an effect typically seen when filamentous microorganisms are observed under the scanning electron microscope. Such flattening might also be caused by the low pressure effects resulting from residence of the filaments in the stratosphere. The

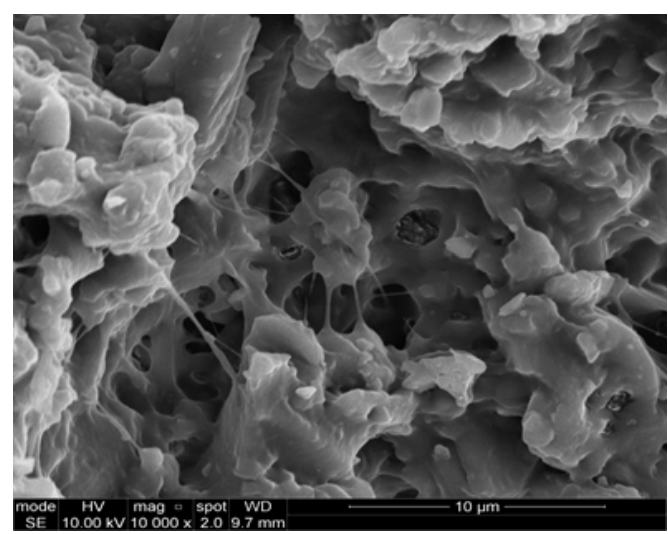

Figure 4a: Detail of a large particle mass isolated from the stratosphere showing microbial filaments, some of which show branching.
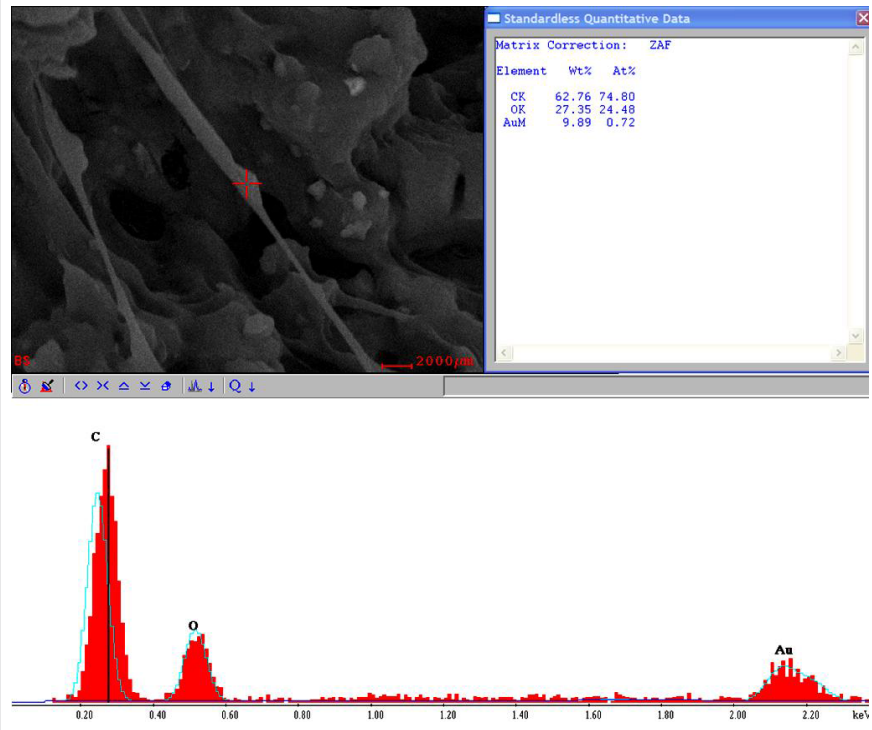

Figure 4b: EDX analysis of a stratosphere-filament; note that the filament is collapsed showing it is a tube and not a solid particle. 


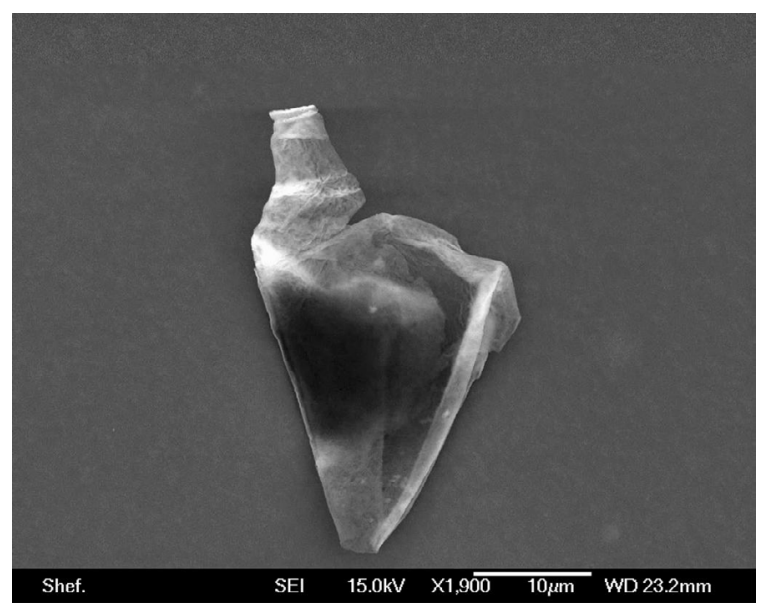

Figure 5: An unknown biological entity isolated from the stratosphere.

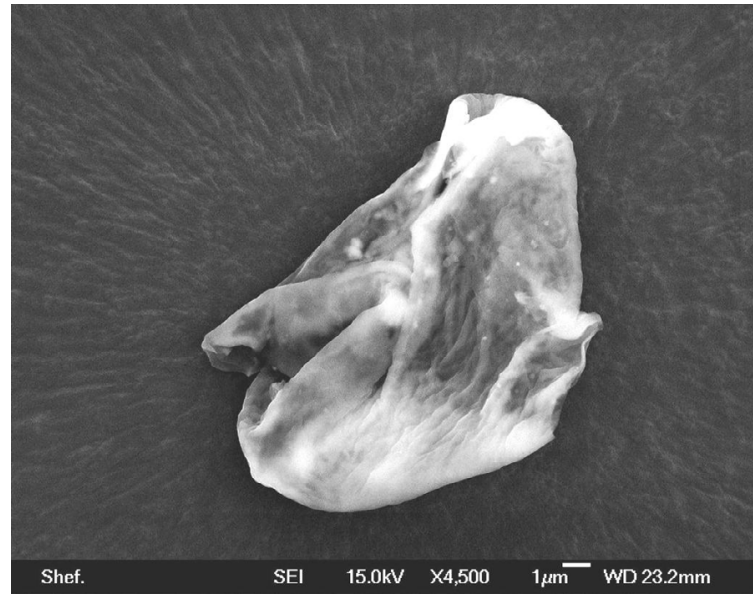

Figure 6: collapsed balloon-like biological entity sampled from the stratosphere. Note the "proboscis" to the left, with nose-like openings and the "sphincter" present at the top of the organism.

obvious conclusion which can be drawn from Figures $4 \mathrm{a}$ and $\mathrm{b}$ is that the stratospheric particle contains a filamentous organism which could be a fungus or a filamentous bacterium, e.g. Nocardia. Although viable fungi have been isolated from the stratosphere and have been grown and identified in the laboratory, this is the first report of filamentous microorganism being observed in situ in a particle isolated from the stratosphere, and which could only have fallen from above.

Another indisputably biological structure that was found on the stubs is shown in Figure 5. Here we see a complex organism which has a segmented neck attached to a flask-shaped body which is ridged and has collapsed under the vacuum of the stratosphere or during $\mathrm{E} / \mathrm{M}$ analysis. The top of the neck is fringed with what could be cilia or a fringe which formed the point of attachment of the neck to another biological entity. The complexity of this particle excludes the possibility that is non-biological in origin. It is clearly too large to be bacterium, but could be an algae or a protozoan; it appears however, too small to be an angiosperm seed, and the fact that the main body has a thin wall appears to exclude the possibility of it being a pollen grain. Pollen grains generally have rigid outer coats that do not collapse when exposed to low pressure.
The structure shown in Figure 6 is also clearly biological in nature; here we see a somewhat phallic balloon-like structure which has presumably collapsed under low pressure. A "proboscis" is seen emerging from the left of the main cell which has two, nostril-like openings. At the top of the collapsed "balloon" is a sphincter-like opening. Again, this entity is clearly biological in nature, and is not an inorganic artefact. Although it is clearly not a bacterium it could well be an alga or a protozoan of some kind.

Finally Figure 7 shows a part of a diatom which, we assume, is clear enough for experts on diatom taxonomy to precisely identify. Similarities with a broad class of diatoms belonging to the Nitzschia species point to a probable tentative identification with a terrestrially known similar organism. The diatom in Figure 7 is obviously incomplete and probably does not contain a protoplast, and is therefore part of an empty frustule. It is not known if the diatom reached the stratosphere as a fragment or whether it arrived with a viable protoplast encased within an intact frustule. The frustule was associated, on the same stub, with cosmic dust particles similar to those seen during balloon sampling flights to $41 \mathrm{~km}[20]$.

\section{Discussion}

Á major problem that faces anyone researching the microbiology of the stratosphere is the difficulty of distinguishing between biological entities, particularly bacteria and fungi, from particles of cosmic dust which in some cases can mimic the morphology of these entities, notably bacteria [20-22]. While structures such as diatom frustules can be readily and unequivocally ascribed to being biological, in the case of bacteria such certainty is rarely forthcoming. This is because structures seen on electron micrographs as naked cocci, or rods are often difficult to distinguish from some types of cosmic dust particles. The use of EDX analysis is of limited value since it cannot identify biological compounds. Moreover stratospheric microbes are often associated with or attached to inorganic particles and the resolution of spot EDX is not always precise. The compositions and morphologies of the structures recovered from $22-27 \mathrm{~km}$ altitude leave little room for doubting their biological provenance, however. The large sizes of the structures, typically several micrometres, give us confidence to propose that they are of extraterrestrial origin, falling from above rather than drifting from below. This assertion is further confirmed by the presence of the craters on the EM stubs shown in Figure 2 and 3c.

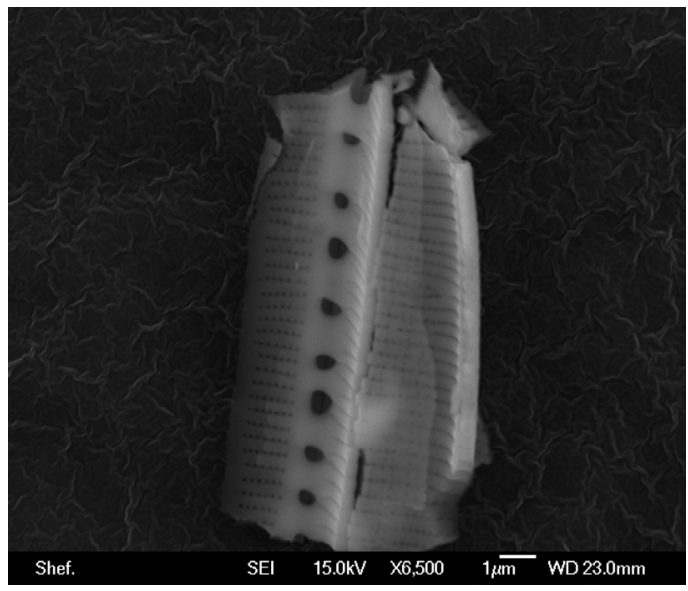

Figure 7: SEM of a diatom frustule (possibly a Nitzschia species) captured on a stub from a height of $25 \mathrm{~km}$ in the stratosphere. 


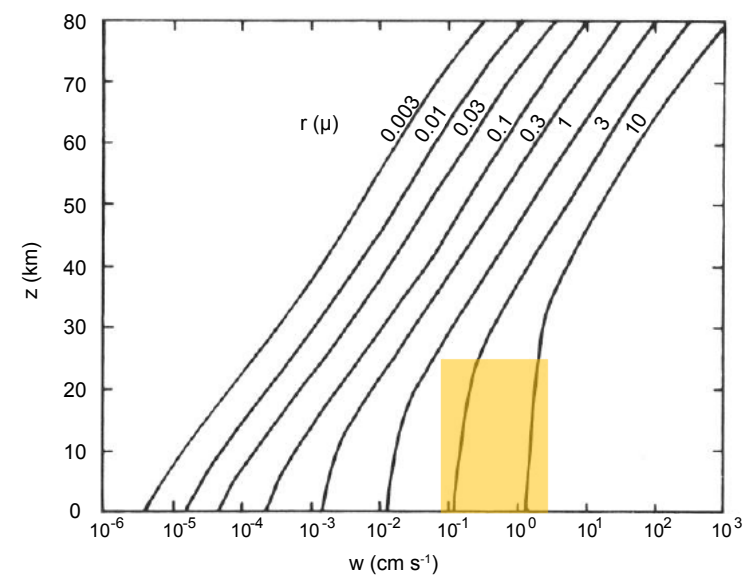

Figure 8: The falling speed of aerosols of various radii in the atmosphere calculated by Kasten (1968). The highlighted domain corresponds to falling diatoms.

There are two possibilities to explain how biological entities of radii in excess of a few micrometres reached the stratosphere; either it was lofted from Earth's surface, or it was falling to Earth from space. Occam's razor might suggest to some that the latter possibility occurred and that they were in some way lofted from Earth to the stratosphere. The most obvious means by which a particle of the size and mass of structures in Figures 3-7, including the diatom fragment, could be lifted from Earth's surface to, say $25 \mathrm{~km}$, is by a violent volcanic eruption. However, no such eruption appears to have taken place in the months prior to the short period of our stratosphere sampling. Even if this happened the residence time in the stratosphere of such relatively large and dense particles would have been very short.

Figure 8 shows the falling speed of spherical particles of various radii (and density $1 \mathrm{~g} \mathrm{~cm}^{-3}$ ) through the atmosphere calculated by Kasten [23] using a standard atmosphere model. From this data we calculate that a particle of radius in the range 3-10 micron would fall at an average speed of $\mathrm{cm} \mathrm{s}^{-1}$ at a height of $20 \mathrm{~km}$, and that the residence time of any particle lofted to this height is about 6 hours; larger particles would have even shorter residence times. The conclusion is that even if a major volcanic eruption occurred a few days before the sampling event, no particles of sizes greater than several microns resulting from it would have been retained in the stratosphere at the point of sampling. There is no record that any such eruption took place. In fact, the most recent major volcanic activity occurring close to the UK was the Evjafjallajokull volcano, which erupted in Iceland in early 2010 and caused considerable problems to commercial flights. There is certainly no way, as is often casually suggested by unthinking critics, that a particle, like the ones seen in Figure 2-6, could simply float into the stratosphere or be carried by winds up to heights well above the tropopause. It is also unlikely that the fragments could have come from commercial aircraft, which fly below well below our stratospheric sampling height. It is noticeable that the diatom fragment in Figure 6 is remarkably clean and free of soil or other solid material, thereby suggesting that it originated from a watery environment, rather than being associated with volcanic debris; in relation to an Earth origin it could have resided in the oceans, while a comet could have provided a cosmic origin for the diatom fragment.

\section{References}

1. Hoyle F, Wickramasinghe NC (1981) Evolution from Space, J.M. Dent, London
2. Hoyle F, Wickramasinghe NC (1985) Living Comets. Cardiff Press, UK

3. Hoyle F, Wickramasinghe NC (2000) Astronomical Origins of Life: Steps towards Panspermia. Kluwer Academic Press, Netherlands.

4. Griffin DW (2004) Terrestrial microorganisms at an altitude of $20,000 \mathrm{~m}$ in Earth's atmosphere. Aerobiologia 20: 135-140.

5. Griffin DW (2008) None-spore forming eubacteria isolated at an altitude of $200,000 \mathrm{~m}$ in Earth's atmosphere: extended incubation periods needed for culture based assays. Aerobiologia 24: 1573-3025

6. Attard E, Yang H, Delort AM, Amato P, Pöschl U, et al. (2012) Effects of atmospheric conditions on ice nucleation activity of Pseudomonas. Atmos. Chem. Phys. 12: 10667-10677.

7. Jayaweera K, Flanagan $P$ (1982) Investigation on biogenic ice nuclei in the Arctic atmosphere, Geophys Res Lett 9: 94-97.

8. Smith DJ, Griffin DW, Schuerger AC (2010) Stratospheric microbiology at 20km over the Pacific Ocean. Aerobiologia 26: 35-46.

9. Bigg EK (1983) In Fundamental Studies and the Future of Science. University College Cardiff Press, Uk.

10. Greene VW, Pederson PD, Lundgren DA, Hagberg CA (1964) Microbiologica exploration of stratosphere: results of six experimental flights. In Proceedings of the Atmospheric Biology Conference, University of Minnesota, Washington USA.

11. Burch CW (1967) Microbes in the upper atmosphere and beyond. Symp Soc Gen Microbiol 17: 345-373.

12. Narlikar JV, Rajaratnam P, Turner MP, Al-Mufti S,Wallis MK, et al. (2001) The detection of living cells in stratospheric samples.

13. Harris MJ, Wickramasinghe NC, Lloyd D, Narlikar JV, Michael PT, et al (2002). Detection of living cells in stratospheric samples. Proceedings of SPIE Conference 4495: 192-198.

14. Wainwright M, Wickramasinghe NC, Narlikar JV, Rajaratnam P (2003) Microorganisms cultured from stratospheric air samples obtained at $41 \mathrm{~km}$ FEMS Microbiol Lett 218: 161-165

15. Yang Y, Itahashi S, Yokobori S, Yamagishi A (2008) UV-resistant bacteria isolated from upper troposphere and lower stratosphere. Biol. Sci.Space 22: 18-25.

16. Yang Y, Yokobori S, Yamagishi A (2009) Assessing panspermia hypothesis by microorganisms collected from the high altitude atmosphere. Biol. Sci. Space 23: 151-163.

17. Shivaji S, Chaturvedi P, Begum Z, Pindi PK, Manorama R, et al. (2009) Janibacter hoylei sp. nov., Bacillus isronensis sp. nov. and Bacillus aryabhatta sp. nov., isolated from cryotubes used for collecting air from the upper atmosphere.Int J Syst Evol Microbiol 59: 2977-2986.

18. Hoyle F, Wickramasinghe NC (1981) In Comets and the Origin of Life

19. Wainwright M, Rose CE, Baker AJ, Bristow KJ, Wickramasinghe NC (2013) Isolation of biological entities from the stratosphere $(22-27 \mathrm{~km})$. J. Cosmol 22 10189-10196.

20. Wainwright M (2008) The high cold biosphere. Focus-Proceedings of the Royal Microscopical Society 12: 32-41.

21. Wainwright M, Wickramasinghe NC, Narlikar JV, Rajaratnam P, Perkins J (2004) Confirmation of the presence of viable but non-cultureable bacteria in the stratosphere. Int. J. Astrobiol. 3: 13-15.

22. Wainwright M, Alharbi S, Wickramasinghe NC (2006) How do microorganisms reach the stratosphere? Intern.J. Astrobiol. 5: 13-16.

23. Kasten F (1968) Falling speed of aerosol particles. J. Appl. Meteorol 7: 944-947 